\title{
Chuvas intensas para o estado de Sergipe com base em dados desagregados de chuva diária
}

\author{
Ricardo de Aragão', Geizon R. de Santana ${ }^{1}$, Clarissa E. F. F. da Costa', \\ Marcus A. S. Cruz ${ }^{2}$, Eduardo E. de Figueiredo ${ }^{3}$ \& Vajapeyam S. Srinivasan ${ }^{4}$
}

\begin{abstract}
RESUMO
A frequência de inundações tem crescido em diversas partes do Brasil e do mundo, como consequência de impactos antrópicos ou de mudanças climáticas. Para mitigar os efeitos das inundações, obras hidráulicas são projetadas com vazão de projeto que pode ser determinada através do emprego de estatística aplicada a séries históricas de vazão ou de precipitação, via equação de chuvas intensas (IDF). Para a determinação da IDF são utilizados dados pluviográficos ou dados pluviométricos diários desagregados. Dada à escassez, no Estado de Sergipe, de dados pluviográficos como também de equações IDF foram desenvolvidas, neste trabalho, as equações IDF para todo o Estado, utilizando-se dados de chuva diária de 48 postos, desagregados com base em fatores de proporcionalidade, além das distribuições de Weibull e Gumbel. Obtiveram-se os melhores resultados com a distribuição de Weibull. A espacialização dos parâmetros da função IDF evidenciou a grande variabilidade das precipitações no Estado de Sergipe.
\end{abstract}

Palavras-chave: enchentes, vazão de projeto, função IDF

\section{Intense rainfall for the State of Sergipe based on disaggregated daily rainfall data}

\begin{abstract}
Floods have frequently been observed worldwide, particularly in urban and rural areas of Brazil as a consequence of antropic impacts and climate change. To cope with the problem hydraulic structures are designed based on the observed maximum flood, or statistically predicted through the rainfall-duration relationship established with recorded data, which are generally scarce. Due to the lack of such data, disaggregation of daily rainfall is generally utilized. In the State of Sergipe-Brazil, the rainfall-duration relationships were established utilizing disaggregated daily rainfalls, observed in 48 stations, and the probability distributions of Weibull and Gumbel. The best results were obtained with the Weibull distribution. The spatial distributions of the parameters of the rainfall-duration relationships demonstrated the high rainfall variability in the region.
\end{abstract}

Key words: flood, design discharge, IDF curves

\footnotetext{
'DEC/UFS, Av. Marechal Rondon, s/n, Jardim Rosa Elze, CEP 49100-000, São Cristóvão, SE. Fone: (79) 2105-6700. E-mail: ricardoaragao@yahoo.com, geizonpe@hotmail.com, clarissa@hotmail.com

${ }^{2}$ Embrapa Tabuleiros Costeiros, Av. Beira Mar, 3250, Jardins, CEP 49025-040, Aracaju, SE. Fone: (79) 4009-1339. E-mail: marcus.cruz@embrapa.br ${ }^{3}$ UAEC/UFCG, Av. Aprígio Veloso 882, Bodocongó, CEP 58429-140, Campina Grande, PB. Fone: (83) 2101-1156. E-mail: eneasef@yahoo.com

${ }^{4}$ CAA/UFPE, Rodovia BR 104, km 59, s/n, Sítio Juriti, Zona Rural, CEP 55002-970, Caruaru, PE. Fone: (81) 2126-8000. E-mail: vajapeyam@yahoo.com
} 


\section{INTRODUÇÃO}

A frequência de inundações nas áreas urbanas e rurais em diversas partes do Brasil e do mundo (Mendes \& Mediondo, 2007; Grimm, 2011) tem aumentado devido às ações antrópicas e às mudanças climáticas que afetam a intensidade das chuvas (Lambert et al., 2008; Min et al., 2011) e aos escoamentos gerados. No meio rural as chuvas intensas e as inundações removem o solo superficial, reduzem sua produtividade, transportam os sedimentos para os corpos hídricos e comprometem sua qualidade e capacidade de armazenamento (Wei, 2007; Santos et al., 2010). No meio urbano levam à destruição de bens e perdas humanas (MCT/CGE, 2002).

Para mitigar os efeitos das inundações obras hidráulicas, como vertedores, canais, barragens e sistemas de drenagem, são projetadas com base na vazão máxima (Teixeira et al., 2011) seja observada estatísticamente ou com base no emprego de modelos chuva-vazão (Mello et al., 2003; Damé et al., 2010). Em pequenas bacias o método racional, que depende da intensidade máxima da chuva (Teixeira et al., 2011) é bastante utilizado. A intensidade máxima da chuva é obtida a partir da relação intensidade-duração-frequência - IDF (Aragão et al., 2000; Ben-Zvi, 2009; Overeem, 2008; Garcia et al., 2011) e sua determinação depende de registros de chuva cuja duração nem sempre é disponível (ANA, 2011) haja vista que precisam de análise cuidadosa (Svensson et al., 2007).

Alternativamente, dados diários podem ser desagregados a partir de fatores de proporcionalidade (CETESB, 1986; Garcia et al., 2011) ou utilizados via método de Bell (Mello, 2003) que se baseia na similaridade entre os mecanismos de tormenta cujos resultados podem ser generalizados. Segundo Mello et al. (2003), Oliveira et al. (2008) e Back (2009), os resultados com dados desagregados se ajustam melhor aos das relações IDF ajustadas com dados de pluviogramas do que as obtidas com o método de Bell. Porém as IDFs são desenvolvidas para um local e precisam de atualização com novos dados (Berne et al., 2004; Damé et al., 2010; Min et al., 2011).

Em Sergipe, no nordeste do Brasil, os dados de chuvas intensas são poucos e antigos (Aragão et al., 2011; SEMARH, 2011) uma vez que só existem para a cidade de Aracaju (CETESB, 1986), os quais foram coletados até 1956. Neste sentido o presente trabalho tem como objetivo o desenvolvimento de equações de chuvas intensas (curvas IDF) para os municípios do Estado de Sergipe através do emprego da metodologia de desagregação de chuvas diárias (CETESB, 1986; Garcia et al., 2011).

\section{Material e Métodos}

O estado de Sergipe, cuja área é cerca de $22.000 \mathrm{~km}^{2}$, está localizado na região Nordeste do Brasil, entre os paralelos $9^{\circ}$ $31^{\prime} 54^{\prime \prime}$ e $11^{\circ} 34^{\prime} 12^{\prime \prime}$ de latitude Sul e os meridianos $36^{\circ} 24^{\prime} 27^{\prime \prime}$ e $38^{\circ} 11^{\prime} 20^{\prime \prime}$ de longitude a Oeste de Greenwich e tem, como limites, os estados da Bahia (ao sul e a oeste) e Alagoas e, a leste, o Oceano Atlântico. A população totaliza 2.068.017 habitantes distribuídos em 75 municípios (IBGE, 2011) dos quais 73,5\% desta população residem na área urbana e 26,5\% na área rural.
O estado tem, como característica climática, a irregularidade espacial e temporal com secas $(47,26 \%$ de seu território estão dentro do polígono das secas) severas em alguns anos e enchentes em outros anos. Esta condição permite classificar o clima (Figura 1) em semiárido (precipitação média de $\sim 700$ $\mathrm{mm}$ ano ${ }^{-1}$ ), agreste (região tropical sub-úmida ou intermediária mais seca; precipitação média de $\sim 1.000 \mathrm{~mm}^{\text {ano }}{ }^{-1}$ ) e litoral úmido (precipitações médias superiores a $\sim 1.355 \mathrm{~mm}^{\mathrm{ano}} \mathrm{o}^{-1}$; umidade relativa do ar com média anual de $80 \%$ ). Nessas regiões a precipitação é concentrada em determinada época do ano definindo uma estação seca e uma chuvosa. O período chuvoso é de abril a agosto, com máximas se concentrando em maio, junho e julho. A temperatura média anual do ar varia de 22,7 a $26,5^{\circ} \mathrm{C}$. A umidade relativa média anual varia de $80 \%$ no Litoral Sudeste a $65 \%$ no noroeste do estado. O mês com menor umidade relativa é o mês de fevereiro (entre 60 a 70\%) e o mês com maior valor é maio (entre 75 a $85 \%$ ) (Aragão et al., 2011; SEMARH, 2011).

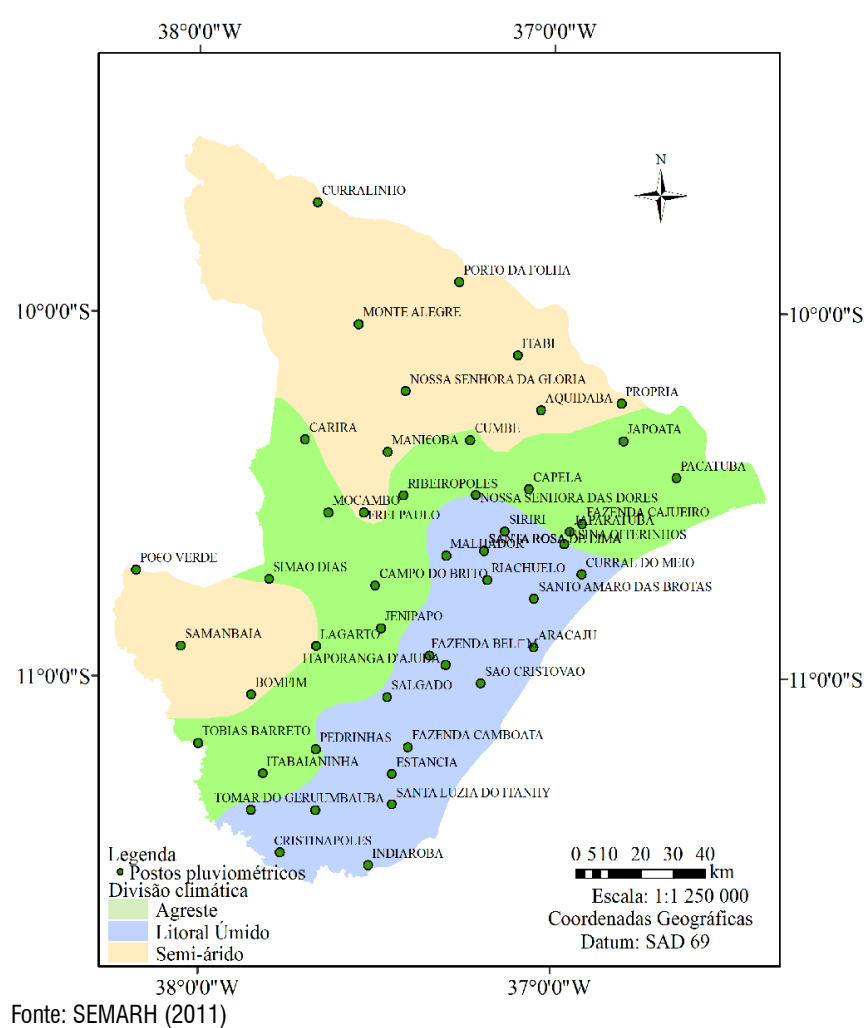

Figura 1. Localização dos postos pluviométricos e divisão climática do estado de Sergipe

\section{Metodologia e dados utilizados na sua aplicação}

A intensidade de uma precipitação qualquer é a relação entre a altura precipitada e sua duração. Para fins de estimativa da vazão máxima a intensidade máxima da precipitação é a variável de interesse sendo esta referida ao tempo de concentração da bacia e associada a um período de retorno. Pode ser obtida mediante as relações intensidade-duração-frequência (IDF), (Eq. 1) estabelecidas a partir de registros de chuva e duração advindos de dados pluviógraficos (Silva et al., 2002).

$$
\mathrm{i}=\frac{\mathrm{K} \cdot \mathrm{T}_{\mathrm{r}}^{\mathrm{a}}}{(\mathrm{t}+\mathrm{B})^{\mathrm{c}}}
$$


em que:

$\begin{array}{ll}\mathrm{i} & \text { - intensidade máxima, } \mathrm{mm} \mathrm{h}^{-1} \\ \mathrm{~T}_{\mathrm{r}} & \text { - período de retorno, anos } \\ \mathrm{t} & \text { - duração da chuva, min } \\ \mathrm{a}, \mathrm{B}, \mathrm{c}, \mathrm{K} \text { - parâmetros a determinar }\end{array}$

Para determinado local a determinação dos parâmetros da equação requer informações de intensidade de chuvas, geralmente escassas. $\mathrm{Na}$ ausência desses dados foi sugerido o método da desagregação de chuva diária (Damé et al., 2010) que relaciona a chuva de 1 dia (observada em pluviômetro) com a chuva de $24 \mathrm{~h}$ (obtida no pluviógrafo) e desta para tempos menores através do emprego de coeficientes (Tabela 1). Também foi considerado, para cada duração citada, um valor limite mínimo de total precipitado a partir do qual a chuva pode ser considerada intensa (Tabela 2), Silva (2009).

Tabela 1. Coeficientes de desagregação para diferentes durações de chuva

\begin{tabular}{cc}
$\begin{array}{c}\text { Relação entre alturas } \\
\text { pluviométricas }\end{array}$ & $\begin{array}{c}\text { Coeficiente de } \\
\text { desagregação }\end{array}$ \\
5 min para 30 min & 0,34 \\
10 min para 30 min & 0,54 \\
15 min para 30 min & 0,70 \\
20 min para 30 min & 0,81 \\
25 min para 30 min & 0,91 \\
30 min para $1 \mathrm{~h}$ & 0,74 \\
1 h para $24 \mathrm{~h}$ & 0,42 \\
2 h para $24 \mathrm{~h}$ & 0,48 \\
3 h para $24 \mathrm{~h}$ & 0,54 \\
6 h para $24 \mathrm{~h}$ & 0,72 \\
$8 \mathrm{~h}$ para $24 \mathrm{~h}$ & 0,78 \\
$10 \mathrm{~h}$ para $24 \mathrm{~h}$ & 0,82 \\
$12 \mathrm{~h}$ para $24 \mathrm{~h}$ & 0,85 \\
$24 \mathrm{~h}$ para $1 \mathrm{dia}$ & 1,14 \\
\hline
\end{tabular}

Fonte: CETESB (1986)

Para o emprego do método da desagregação de chuva fazse necessário separar, inicialmente, os valores máximos de precipitação para cada ano do posto em questão; em seguida foram aplicados, a cada valor de precipitação, os coeficientes listados na Tabela 1 gerando, assim, séries de precipitação para diferentes durações. Com base nos valores da Tabela 2 foram eliminados da série os valores de precipitação abaixo de $8 \mathrm{~mm}$ com duração de 5 min.

Para as séries com outras durações (Tabela 2) foi considerado o mesmo número de anos (elementos da série) previamente escolhidos, para 5 min de duração garantindo, então, que os valores da série de precipitação fossem, no mínimo, iguais aos valores listados na Tabela 2.

De acordo com CETESB (1986) nos estudos hidroclimatológicos poderão ser utilizadas séries anuais (que incluem a altura pluviométrica máxima de cada ano), bem como séries parciais (constituídas pelos n maiores totais precipitados, sendo $\mathrm{n}$ o número de anos ou o número de elementos da série, independente do ano em que possam ocorrer). A escolha do tipo da série depende do seu tamanho e do objetivo do estudo. As séries parciais fornecem resultados mais consistentes para número de anos de dados menores que 12 anos. Além disto, praticamente as duas séries contemplam os mesmos resultados para períodos de retorno superiores a 10 anos (Teixeira et al., 2011).

Neste trabalho foram aplicados, utilizando-se planilha eletrônica, os coeficientes listados nas Tabelas 1 e 2 aos dados de séries anuais. Nos casos em que, após o processo de desagregação e aplicação dos coeficientes da Tabela 2 as séries ficaram com menos de 10 anos, séries parciais foram estabelecidas com os n maiores valores de precipitação máxima e novamente o processo de desagregação foi aplicado.

Para Naghettini \& Pinto (2007) nos trabalhos hidrológicos, além da determinação das máximas precipitações observadas nas séries históricas, faz-se necessário também prever, com o auxílio de probabilidades, a frequência associada a essas precipitações. Neste sentido, a distribuição de Gumbel e a distribuição de Weibull, além da log-Pearson III, se ajustam satisfatoriamente às distribuições de valores extremos de grandezas hidrológicas, tais como totais precipitados e vazões observadas sendo, assim, empregadas neste trabalho (Naghettini \& Pinto, 2007; Sansigolo, 2008).

A distribuição de Gumbel apresenta a seguinte função cumulativa de probabilidade:

$$
\text { FCP }: P[Y \leq y]=\mathrm{e}^{-\mathrm{e}^{-\alpha(y-\mu)}}
$$

em que:

P - probabilidade de não excedência;

$\alpha, \mu$ - parâmetros da distribuição

y - variável reduzida

O parâmetro $\alpha$ é o de escala e o parâmetro $\mu$ de locação. Por meio do método dos momentos e com as estimativas amostrais $\mathrm{x}$ (média aritmética) e $\mathrm{S}$ (desvio padrão), tem-se, como resultado:

$$
\begin{gathered}
\mathrm{z}=\alpha(\mathrm{y}-\mu) \\
\alpha=\frac{1,2826}{\mathrm{~S}} \\
\mu=\overline{\mathrm{x}}-0,451 \mathrm{~S} \\
\mathrm{y}=\left[\mathrm{x}-\overline{\mathrm{x}}+\mathrm{S}\left(\frac{\mathrm{yn}}{\mathrm{Sn}}\right)\right] \frac{\mathrm{Sn}}{\mathrm{S}}
\end{gathered}
$$

Substituindo os coeficientes $\alpha$ e $\mu$ na Eq. 3, tem-se a Eq. 7:

\begin{tabular}{|c|c|c|c|c|c|c|c|c|c|c|c|c|c|c|}
\hline Duração (min) & 5 & 10 & 15 & 20 & 30 & 45 & 60 & 120 & 180 & 240 & 360 & 480 & 720 & 1440 \\
\hline Precipitação adotada (mm) & 8 & 10 & 15 & 15 & 20 & 23 & 25 & 30 & 33 & 35 & 40 & 40 & 47 & 55 \\
\hline
\end{tabular}

$$
\begin{gathered}
\mathrm{x}=\overline{\mathrm{x}}+\mathrm{KS} \\
\mathrm{K}=0,7797 \mathrm{z} \times 0,45
\end{gathered}
$$

Tabela 2. Valores mínimos de precipitação, adotados 
em que:

$\mathrm{y}_{\mathrm{n}}$ e $\mathrm{S}_{\mathrm{n}}$ - respectivamente, a média e o desvio padrão da variável reduzida

$\mathrm{T}_{\mathrm{r}} \quad$ - período de retorno $=1 /(1-\mathrm{P}(\mathrm{Y}<\mathrm{y}))$

Segundo Naghettini \& Pinto (2007) e Murthy et al. (2004) a distribuição de Weibull é da mesma família da distribuição de Gumbel porém é chamada distribuição de extremos do tipo III; com larga aplicação em diversas áreas do conhecimento, ajusta-se bem à distribuição de vazões anuais e de precipitação, sendo representada pela seguinte função cumulativa de probabilidade:

$$
\begin{gathered}
\mathrm{FCP}: \mathrm{P}[\mathrm{X} \geq \mathrm{x}]=\mathrm{e}^{-\left[\frac{(\mathrm{x}-\gamma)]^{\lambda}}{(\mu-\gamma)}\right]^{\lambda}} \\
\frac{1}{\lambda}=0,27777579+0,313261774 \mathrm{~g}(\mathrm{x})+ \\
+\quad 0,057567091 \mathrm{~g}(\mathrm{x})^{2}-0,0013038566 \mathrm{~g}(\mathrm{x})^{3}- \\
-0,0081523408 \mathrm{~g}(\mathrm{x})^{4} \\
\mathrm{~g}(\mathrm{x})=\left[\frac{\left[\sum(\mathrm{x}-\overline{\mathrm{x}})^{3}\right]}{\mathrm{N}}\right] \mathrm{S}^{3} \\
\mu=\overline{\mathrm{x}}+\mathrm{s}\left[1-\Gamma\left(1+\frac{1}{\lambda}\right)\right] \sqrt{\left[\Gamma \Gamma\left(+\frac{2}{\lambda}\right)-\Gamma^{2}\left(1+\frac{1}{\lambda}\right)\right]} \\
\gamma=\mu-\mathrm{s} \sqrt{\left[\Gamma \Gamma\left(+\frac{2}{\lambda}\right)-\Gamma^{2}\left(1+\frac{1}{\lambda}\right)\right]}
\end{gathered}
$$

em que:

$\Gamma$ - logaritmo neperiano (natural) da função gama (lngama)

$\mathrm{P}(\mathrm{X}<\mathrm{x})$ - probabilidade de não excedência

$\mathrm{X} \quad$ - média da amostra $\mathrm{S}$ - desvio padrão da amostra

$\mathrm{T}_{\mathrm{r}}$ - período de retorno

$\mathrm{g}(\mathrm{x})$ - estimativa amostral de assimetria

A aderência das séries de máximas às distribuições testadas foi verificada através das seguintes ferramentas estatísticas (Naghetinni \& Pinto, 2007): pelo teste de aderência de Kolmogorov-Sminorv (K-S) a nível de significância de 5\%, pelo coeficiente de determinação $\left(\mathrm{R}^{2}\right)$, pelo coeficiente de ajuste (CA) e pelo coeficiente de massa residual (CMR).

$$
\mathrm{D}_{\mathrm{N}}=\sup _{-\infty<i<\infty}\left|\mathrm{F}_{\mathrm{N}}(\mathrm{Ti})-\mathrm{Mi}\right|
$$

em que:

$$
\begin{gathered}
\mathrm{F}_{\mathrm{N}}\left(\mathrm{T}_{\mathrm{i}}\right)=\mathrm{i} / \mathrm{N} \text { e } 1 \leq \mathrm{i} \leq \mathrm{N} ; \mathrm{KS}=\left|\mathrm{Fi}-\mathrm{M}_{\mathrm{i}}\right| ; \text { onde } \\
\mathrm{Fi}=\frac{\mathrm{m}_{\mathrm{i}}}{\mathrm{n}+1}
\end{gathered}
$$

$$
\begin{gathered}
\mathrm{R}^{2}=\frac{\left[\mathrm{n}\left(\sum \mathrm{M}_{\mathrm{i}} \cdot \mathrm{T}_{\mathrm{i}}\right)\right]^{2}}{\left[\mathrm{n} \sum \mathrm{T}_{\mathrm{i}}^{2}-\left(\sum \mathrm{T}_{\mathrm{i}}\right)^{2}\right] \cdot\left[\mathrm{n} \sum \mathrm{M}_{\mathrm{i}}^{2}-\left(\sum \mathrm{M}_{\mathrm{i}}\right)^{2}\right]} \\
\mathrm{CA}=\frac{\sum\left(\mathrm{M}_{\mathrm{i}}-\overline{\mathrm{M}}\right)^{2}}{\sum\left(\mathrm{T}_{\mathrm{i}}-\overline{\mathrm{M}}\right)^{2}} \\
\mathrm{CMR}=\left[\frac{\sum \mathrm{M}_{\mathrm{i}}-\sum \mathrm{T}_{\mathrm{i}}}{\sum \mathrm{M}_{\mathrm{i}}}\right]
\end{gathered}
$$

em que:

$$
\begin{array}{ll}
M_{i} & \text { - valores calculados pelos modelos } \\
T_{i} & \text { - valores observados das séries históricas }
\end{array}
$$

Empregaram-se os quatro critérios com o objetivo de uma definição melhor da distribuição mais adequada para a série e reduzir, assim, as incertezas inerentes às análises desta espécie.

$\mathrm{O}$ teste K-S avalia a distância máxima entre os resultados de uma distribuição a ser testada e os valores associados à distribuição hipoteticamente verdadeira. O coeficiente CA descreve a razão entre a dispersão dos valores observados e os calculados teoricamente e deverá tender a um. O coeficiente CMR tende a zero na ausência de desvios sistemáticos entre os valores observados e os calculados podendo indicar a superestimação $(C M R>0)$ ou a subestimação $(C M R<0)$ dos valores estimados pelas distribuições teóricas de probabilidade.

Visto que as distribuições de probabilidade foram ajustadas às séries desagregadas, calcularam-se então os valores de precipitação para os períodos de retorno utilizados em projetos de obras hidráulicas (CETESB, 1986): 2, 5, 10, 25, 50, 100 anos. Para cada período de retorno e com as durações da Tabela 2 foram determinadas as intensidades máximas médias. De posse das séries de intensidades foram calculados os parâmetros da Eq.1, via método de regressão linear, logaritmizando a Eq.1, e não-linear via softwares de ajustes de curva, lembrando que o numerador da Eq.1 é não linear, sendo prudente testar a melhor regressão. Para o emprego dos dois métodos foi utilizado o software Lab-fit ajuste de curvas V7.2.19 (Silva et al., 2004). Neste software o critério mais utilizado para definir o melhor ajuste se baseia no menor qui-quadrado reduzido para a função ajustada. Foi testado, via $\mathrm{R}^{2}$ e erro padrão da Estimativa (EPE) (Eq.19), qual dos dois métodos leva aos melhores resultados e também ao grau de precisão dos modelos utilizados para determinação da equação de chuvas intensas.

$$
\mathrm{EPE}=\sqrt{\frac{\sum_{\mathrm{i}=1}^{\mathrm{n}}\left(\frac{\mathrm{Ic}-\mathrm{Io}}{\mathrm{Io}}\right)^{2}}{\mathrm{~N}}}
$$

em que:

Ic - intensidade $\left(\mathrm{mm} \mathrm{h}^{-1}\right)$ calculada por meio da equação IDF

Io - intensidade $\left(\mathrm{mm} \mathrm{h}^{-1}\right)$ extraída do melhor ajuste de distribuição

$\mathrm{N}$ - número de durações 
Utilizaram-se dados de postos pluviométricos no estado de Sergipe e disponíveis na Hidroweb - Sistema de Informações Hidrológicas (ANA, 2011). Dos 129 postos existentes (ativos ou inativos) 71 foram selecionados (Figura 1) distribuídos pelo território sergipano, com séries mais longas e contínuas possíveis; para tanto, eliminaram-se das séries os anos nos quais havia falhas diárias em virtude da dificuldade de preenchê-las; devido ao grande número de postos não foi possível listar os intervalos em que a falha diária foi encontrada. Também foram utilizados, no presente estudo, os dados dos postos Aracaju, Itabaianinha e Própria, monitorados pelo Instituto Nacional de Meteorologia (INMET) nas respectivas cidades.

\section{Resultados e Discussão}

Foram geradas, a partir dos dados dos 74 postos selecionados, as séries históricas de precipitação máxima diária e aplicada a metodologia de desagregação de chuva a partir dos coeficientes listados nas Tabelas 1 e 2 . Para 26 postos e mesmo se considerando séries parciais, as séries históricas ficaram com um número bem inferior a cinco anos de dados após o processo de desagregação, tendo sido descartados do estudo.

Para os 48 postos restantes foram formadas séries anuais em 39 postos e parciais em 9 postos, com as quais foi possível efetuar a análise de frequência e a previsão de valores extremos a partir das distribuições investigadas, tal como a determinação dos parâmetros da Eq.1 (Figuras 2 e 3; Tabela 3). Devido à eliminação dos anos com falhas o número de anos utilizados para o estudo é, em alguns casos, bem menor que o período listado na Tabela 3.

Após a aplicação das distribuições de Gumbel e Weibull e se considerando o coeficiente de determinação $\left(\mathrm{R}^{2}\right)$, o teste K-S, o coeficiente de ajuste (CA) e o coeficiente de massa residual (CMR) verificou-se que houve tendência em 44 postos de

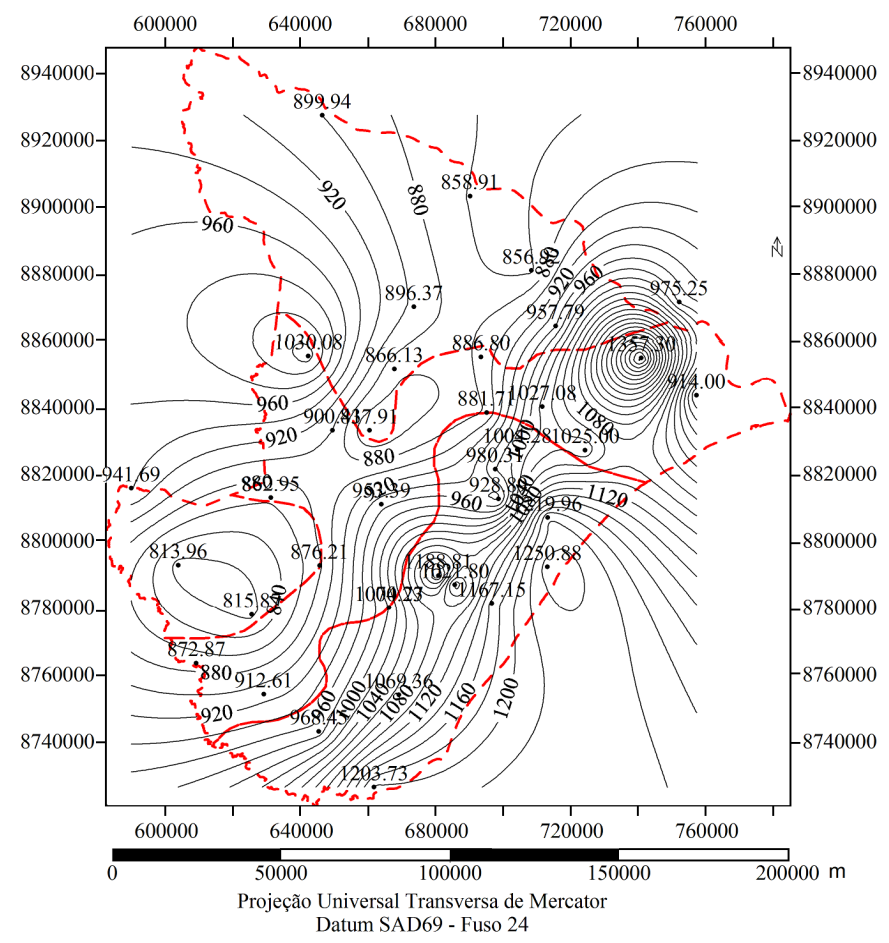

Figura 2. Espacialização dos valores do coeficiente $\mathrm{K}$

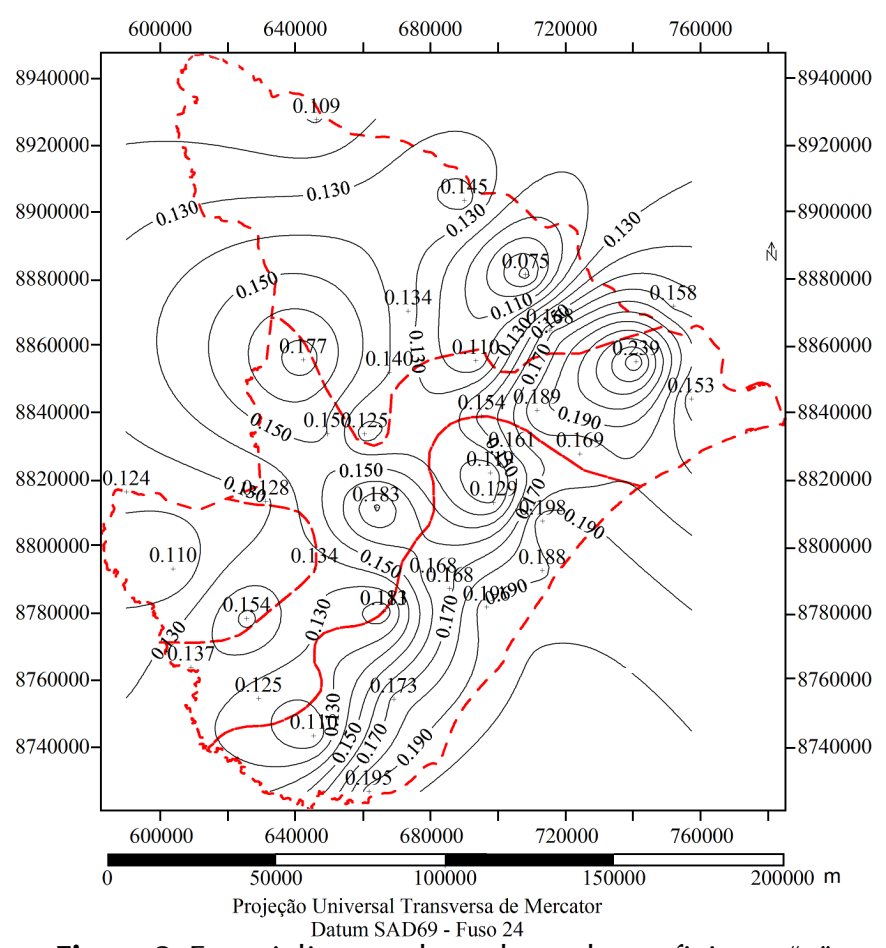

Figura 3. Espacialização dos valores do coeficiente "a"

melhor aderência dos dados à distribuição de Weibull (Tabela 4). A distribuição de Weibull também foi a que melhor se adequou aos dados de chuvas intensas nas estações no estado de Pernambuco (Silva, 2009) geradas com a mesma metodologia aqui empregada (desagregação de chuva de 1 dia). Pelos mesmos critérios a distribuição de Gumbel se adequou melhor aos dados dos postos Aracaju, Itaporanga d'Ajuda, Siriri e Tomar do Geru.

Os resultados obtidos foram diferentes do que a literatura comenta sobre as distribuições de probabilidades aplicadas a valores extremos máximos, em que a distribuição de Gumbel (Naghettine \& Pinto, 2007; Ben-Zvi, 2009) surge como a mais indicada e melhor ajustada. Overeem et al. (2008) alertam para o fato de que, apesar de bastante utilizada, a distribuição de Gumbel pode subestimar as quantidades para longos períodos de retorno. No entanto, é de se esperar que a distribuição de Weibull se adapte bem, tanto às frequências com valores máximos quanto mínimos, fato que foi comprovado através do presente estudo e no trabalho de Silva (2009) e Murthy et al. (2004); assim e com base nos resultados obtidos, a distribuição de Weibull poderá também ser aplicada aos estudos de chuvas intensas.

Após a determinação dos parâmetros da Eq.1 via método de regressão linear e não-linear, verificou-se aderência entre os dados gerados pelas equações ajustadas e as séries de intensidades provenientes das distribuições de probabilidade para os dois métodos.

Devido ao volume de dados não é possível a apresentação de todos os valores dos parâmetros para as duas regressões (linear e não linear) porém é possível resumir os resultados da seguinte maneira: o valor do coeficiente de determinação $\mathrm{R}^{2}$ permaneceu constante e igual a 0,99 ; os valores da EPE (erro padrão da estimativa) ficaram entre 1 e 3 , tendo sido sempre menores para a regressão não-linear; os valores do CMR (coeficiente de massa residual) tenderam a zero para a regressão 
Tabela 3. Postos pluviométricos selecionados no Estado de Sergipe, SE

\begin{tabular}{|c|c|c|c|c|c|c|c|c|}
\hline Id & Cód. & Long. & Lat. & Alt.(m) & Posto & Município & Período & $\mathrm{N}^{*}$ \\
\hline 1 & 1037003 & $-37: 02$ & $-10: 16$ & 217 & Aquidabã & Aquidabã & 1913-1991 & 58 \\
\hline 2 & 1037004 & $-37: 03$ & $-10: 55$ & 472 & Aracaju & Aracaju & $1963-2009$ & 43 \\
\hline 3 & 1137011 & $-37: 51$ & $-11: 03$ & 230 & Bomfim & Riachão do Dantas & $1913-1986$ & 70 \\
\hline 4 & 1037008 & $-37: 30$ & $-10: 45$ & 180 & Campo do Brito & Campo do Brito & $1953-2000$ & 30 \\
\hline 5 & 1037078 & $-37: 04$ & $-10: 29$ & 139 & Capela & Capela & 1983-2007 & 22 \\
\hline 6 & 1037010 & $-37: 42$ & $-10: 21$ & 351 & Carira & Carira & $1964-1984$ & 20 \\
\hline 7 & 1137014 & $-37: 46$ & $-11: 29$ & 173 & Cristinápoles & Cristinápoles & $1964-1984$ & 13 \\
\hline 8 & 1037013 & $-37: 14$ & $-10: 21$ & 180 & Cumbe & Cumbe & 1964-1987 & 23 \\
\hline 9 & 1036033 & $-36: 55$ & $-10: 43$ & 30 & Curral do Meio & Sto. Amaro das Brotas & 1964-1984 & 18 \\
\hline 10 & 937029 & $-37: 40$ & $-9: 42$ & 80 & Curralinho & Poço Redondo & $1938-1984$ & 38 \\
\hline 11 & 1137015 & $-37: 27$ & $-11: 16$ & 53 & Estância & Estancia & $1944-2006$ & 43 \\
\hline 12 & 1037007 & $-37: 20$ & $-10: 56$ & 19 & Faz Belém & Itaporanga D'ajuda & 1949-2007 & 49 \\
\hline 13 & 1036063 & $-36: 54$ & $-10: 34$ & 10 & Faz. Cajueiro & Japaratuba & 1993-2004 & 11 \\
\hline 14 & 1137012 & $-37: 30$ & $-11: 08$ & 150 & Faz. Camboatã & Estância & $1963-1984$ & 18 \\
\hline 15 & 1037014 & $-37: 32$ & $-10: 33$ & 272 & Frei Paulo & Frei Paulo & $1913-1980$ & 62 \\
\hline 16 & 1137018 & $-37: 31$ & $-11: 31$ & 21 & Indiaroba & Indiaroba & $1964-1999$ & 23 \\
\hline 17 & 1037021 & $-37: 06$ & $-10: 07$ & 187 & Itabi & Itabi & 1964-1998 & 22 \\
\hline 18 & 1137021 & $-37: 49$ & $-11: 16$ & 208 & Itabaianinha & Itabaianinha & $1963-2009$ & 46 \\
\hline 19 & 1037022 & $-37: 18$ & $-10: 58$ & 10 & Itaporanga d'ajuda & Itaporanga D'ajuda & $1914-1975$ & 57 \\
\hline 20 & 1036014 & $-36: 57$ & $-10: 36$ & 79 & Japaratuba & Japaratuba & 1918-1985 & 61 \\
\hline 21 & 1036018 & $-36: 48$ & $-10: 21$ & 89 & Japoatã & Japoatã & 1964-1997 & 21 \\
\hline 22 & 1037024 & $-37: 29$ & $-10: 52$ & 100 & Jenipapo & Lagarto & 1963-1981 & 16 \\
\hline 23 & 1037061 & $-37: 40$ & $-10: 55$ & 183 & Lagarto & Lagarto & $1921-1996$ & 58 \\
\hline 24 & 1037030 & $-37: 18$ & $-10: 40$ & 224 & Malhador & Malhador & $1964-1984$ & 15 \\
\hline 25 & 1037031 & $-37: 28$ & $-10: 23$ & 250 & Maniçoba & N. Sra. Aparecida & $1964-1999$ & 21 \\
\hline 26 & 1037032 & $-37: 38$ & $-10: 33$ & 204 & Mocambo & Frei Paulo & 1913-1978 & 61 \\
\hline 27 & 1037033 & $-37: 33$ & $-10: 02$ & 230 & Monte Alegre & Monte Alegre & 1964-1997 & 15 \\
\hline 28 & 1037034 & $-37: 25$ & $-10: 13$ & 290 & N. Sra. da Glória & N. Sra. da Glória & 1913-1997 & 71 \\
\hline 29 & 1037036 & $-37: 13$ & $-10: 30$ & 200 & N. Sra. das Dores & N. Sra. das Dores & 1914-1999 & 74 \\
\hline 30 & 1036021 & $-36: 39$ & $-10: 27$ & 20 & Pacatuba & Pacatuba & 1921-1984 & 60 \\
\hline 31 & 1137035 & $-37: 40$ & $-11: 12$ & 170 & Pedrinhas & Pedrinhas & $1944-1960$ & 16 \\
\hline 32 & 1038000 & $-38: 10$ & $-10: 42$ & 300 & Poço verde & Poço Verde & $1938-1984$ & 35 \\
\hline 33 & 937026 & $-37: 16$ & $-9: 55$ & 45 & Porto da Folha & Porto da Folha & 1914-1999 & 61 \\
\hline 34 & 1036025 & $-36: 52$ & $-10: 12$ & 20 & Propria & Propria & $1963-2009$ & 43 \\
\hline 35 & 1037042 & $-37: 11$ & $-10: 44$ & 30 & Riachuelo & Riachuelo & 1964-1997 & 22 \\
\hline 36 & 1037044 & $-37: 26$ & $-10: 32$ & 350 & Ribeirópolis & Ribeirópolis & 1964-1997 & 23 \\
\hline 37 & 1137001 & $-37: 28$ & $-11: 01$ & 92 & Salgado & Salgado & $1948-2006$ & 39 \\
\hline 38 & 1038001 & $-38: 03$ & $-10: 55$ & 250 & Samanbaia & Tobias Barreto & 1963-1997 & 20 \\
\hline 39 & 1137002 & $-37: 27$ & $-11: 21$ & 50 & Sta. Luzia do Itanhy & Sta. Luzia do Itanhy & $1964-1986$ & 19 \\
\hline 40 & 1037049 & $-37: 11$ & $-10: 39$ & 47 & Sta Rosa de Lima & Sta Rosa de Lima & $1957-2006$ & 27 \\
\hline 41 & 1037050 & $-37: 03$ & $-10: 47$ & 9 & Sto. Amaro das Brotas & Sto. Amaro das Brotas & $1964-1998$ & 23 \\
\hline 42 & 1137007 & $-37: 12$ & $-11: 01$ & 20 & São Cristóvão & São Cristóvão & $1964-2000$ & 22 \\
\hline 43 & 1037045 & $-37: 48$ & $-10: 44$ & 283 & Simão Dias & Simão Dias & $1913-1987$ & 69 \\
\hline 44 & 1037047 & $-37: 08$ & $-10: 36$ & 90 & Siriri & Siriri & $1963-1998$ & 24 \\
\hline 45 & 1138000 & $-38: 00$ & $-11: 11$ & 157 & Tobias Barreto & Tobias Barreto & $1914-1981$ & 62 \\
\hline 46 & 1137003 & $-37: 51$ & $-11: 22$ & 120 & Tomar do Geru & Tomar do Geru & $1964-1998$ & 15 \\
\hline 47 & 1137004 & $-37: 40$ & $-11: 22$ & 109 & Umbauba & Umbauba & 1964-1999 & 21 \\
\hline 48 & 1036020 & $-36: 58$ & $-10: 38$ & 80 & Usina Oiterinhos & Japaratuba & $1965-1991$ & 19 \\
\hline
\end{tabular}

* Número de anos de observação usados no trabalho

não-linear; o CA (coeficiente de ajuste) para a regressão nãolinear ficou sempre abaixo dos seus respectivos valores para a regressão linear, levando a crer que este método conduz ao melhor conjunto dos valores dos parâmetros a, B, c, K.

Os parâmetros da Eq. 1 também foram calculados para a distribuição de Gumbel, visando às futuras comparações com valores desenvolvidos para postos nas fronteiras do Estado de Sergipe. Observa-se, na Tabela 4, que o coeficiente a variou de 0,061 (Ribeirópolis) a 0,239 (Japoatã) e o coeficiente $\mathrm{K}$ variou de 778,4 (Bomfim) a 1357,3 (Japoatã). Por outro lado, o erro padrão da estimativa variou entre 1,15 e 3,014 sendo, em média, $4 \%$ menor para os postos cujos parâmetros foram gerados a partir da distribuição de Weibull. Nota-se, assim, uma grande variabilidade dos parâmetros $\mathrm{K}$ e a para as estações, o que denota larga variabilidade de intensidade entre as estações, mesmo entre as mais próximas, fato constatado também por Silva et al. (2002), Aragão et al. (2000) confirmando a alta variabilidade da precipitação e a necessidade de estudos sobre o tema para abranger uma área o maior possível do Estado. O valor de $\mathrm{R}^{2}$ ficou sempre acima de 0,99 sinalizando que este índice pode ser tendencioso e não adequado para avaliar o ajustamento da Eq. 1 aos dados observados.

Por sua vez, a regressão não-linear forneceu valores constantes para os parâmetros B e c $(10,52$ e 0,753 , respectivamente) para todos os postos e para as duas distribuições. Esta tendência, também observada por Oliveira et 
Tabela 4. Valores dos parâmetros para a equação IDF

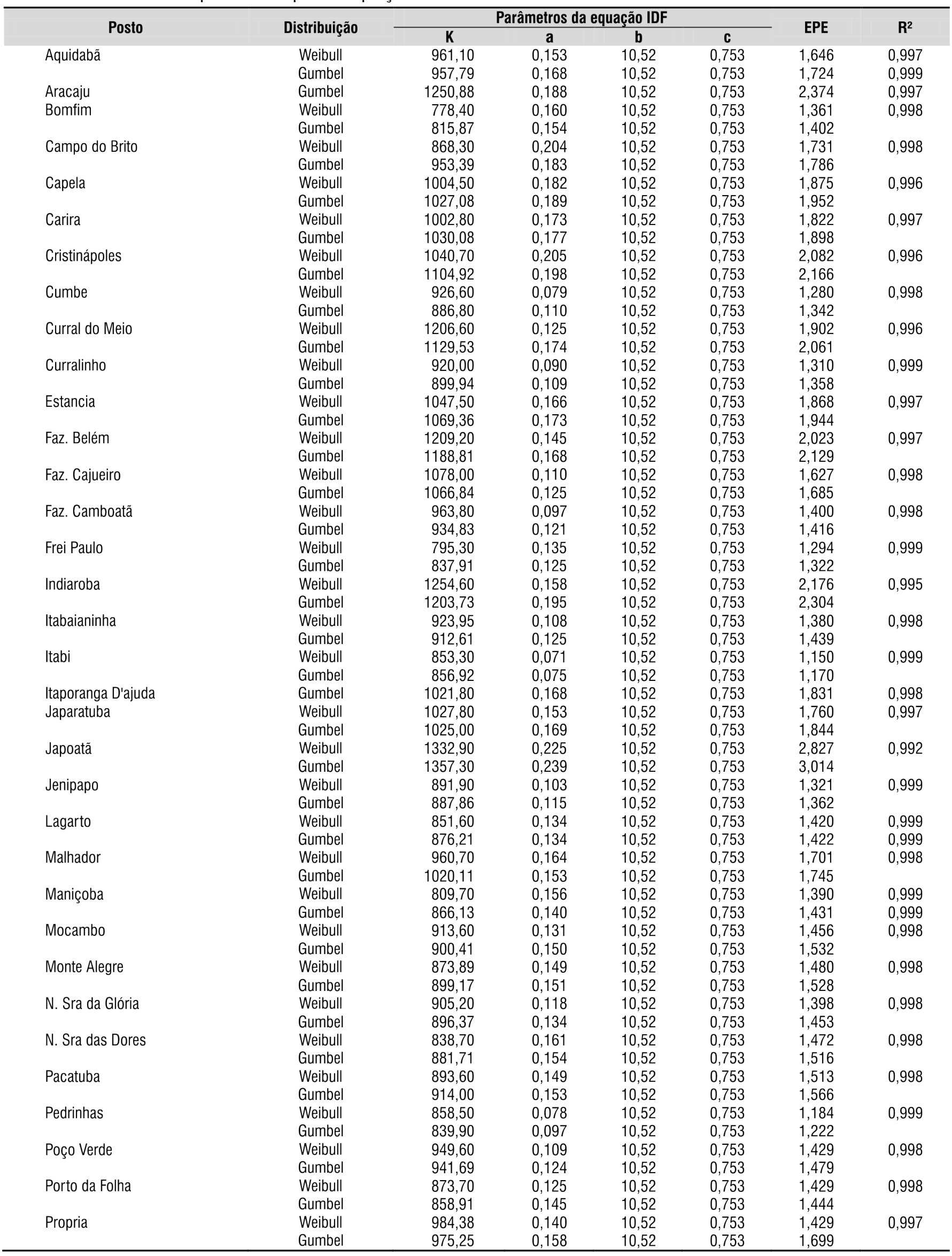


Continuação da Tabela 4

\begin{tabular}{|c|c|c|c|c|c|c|c|}
\hline \multirow{2}{*}{ Posto } & \multirow{2}{*}{ Distribuição } & \multicolumn{4}{|c|}{ Parâmetros da equação IDF } & \multirow{2}{*}{ EPE } & \multirow{2}{*}{$\mathbf{R}^{2}$} \\
\hline & & K & $\mathbf{a}$ & b & C & & \\
\hline \multirow[t]{2}{*}{ Riachuelo } & Weibull & 944,20 & 0,110 & 10,52 & 0,753 & 1,426 & 0,998 \\
\hline & Gumbel & 928,89 & 0,129 & 10,52 & 0,753 & 1,482 & \\
\hline \multirow[t]{2}{*}{ Ribeirópolies } & Weibull & 1080,50 & 0,061 & 10,52 & 0,753 & 1,417 & 0,997 \\
\hline & Gumbel & 1000,23 & 0,113 & 10,52 & 0,753 & 1,525 & \\
\hline \multirow[t]{2}{*}{ Salgado } & Weibull & 1053,30 & 0,173 & 10,52 & 0,753 & 1,915 & 0,997 \\
\hline & Gumbel & 1074,77 & 0,181 & 10,52 & 0,753 & 2,000 & \\
\hline \multirow[t]{2}{*}{ Samanbaia } & Weibull & 821,80 & 0,097 & 10,52 & 0,753 & 1,194 & 0,999 \\
\hline & Gumbel & 813,96 & 0,110 & 10,52 & 0,753 & 1,231 & \\
\hline \multirow[t]{2}{*}{ Sta luzia do Itanhy } & Weibull & 1047,30 & 0,089 & 10,52 & 0,753 & 1,491 & 0,998 \\
\hline & Gumbel & 995,89 & 0,126 & 10,52 & 0,753 & 1,574 & \\
\hline \multirow[t]{2}{*}{ Sta Rosa de Lima } & Weibull & 1022,90 & 0,088 & 10,52 & 0,753 & 1,450 & 0,998 \\
\hline & Gumbel & 980,31 & 0,119 & 10,52 & 0,753 & 1,522 & \\
\hline \multirow[t]{2}{*}{ Santo Amaro das Brotas } & Weibull & 1259,90 & 0,165 & 10,52 & 0,753 & 2,234 & 0,995 \\
\hline & Gumbel & 1219,96 & 0,198 & 10,52 & 0,753 & 2,390 & \\
\hline \multirow[t]{2}{*}{ São Cristóvão } & Weibull & 1143,70 & 0,187 & 10,52 & 0,753 & 2,166 & 0,996 \\
\hline & Gumbel & 1167,15 & 0,196 & 10,52 & 0,753 & 2,272 & \\
\hline \multirow[t]{2}{*}{ Simão Dias } & Weibull & 862,50 & 0,117 & 10,52 & 0,753 & 1,330 & 0,998 \\
\hline & Gumbel & 862,95 & 0,128 & 10,52 & 0,753 & 1,375 & \\
\hline Siriri & Gumbel & 1004,28 & 0,161 & 10,52 & 0,753 & 1,758 & \\
\hline \multirow[t]{2}{*}{ Tobias Barreto } & Weibull & 862,90 & 0,131 & 10,52 & 0,753 & 1,380 & 0,998 \\
\hline & Gumbel & 872,87 & 0,137 & 10,52 & 0,753 & 1,428 & \\
\hline Tomar do geru & Gumbel & 1253,01 & 0,178 & 10,52 & 0,753 & 2,380 & \\
\hline \multirow[t]{2}{*}{ Umbauba } & Weibull & 994,50 & 0,088 & 10,52 & 0,753 & 1,409 & 0,999 \\
\hline & Gumbel & 968,45 & 0,110 & 10,52 & 0,753 & 1,409 & \\
\hline \multirow[t]{2}{*}{ Usina Oiterinhos } & Weibull & 1182,90 & 0,117 & 10,52 & 0,753 & 1,821 & 0,996 \\
\hline & Gumbel & 1096,63 & 0,171 & 10,52 & 0,753 & 1,982 & \\
\hline
\end{tabular}

al. (2008) e Silva (2009) leva a crer ser isto uma consequência da desagregação de chuvas diárias, da forma da aplicação da Tabela 2 ou da forma de determinação dos parâmetros da Eq. 1 , uma vez que, para dados de pluviógrafos, a mesma tendência não foi observada (Aragão et al., 2000; Cecílio \& Pruski, 2003; Silva, 2009).

Para esclarecer o motivo dos valores constantes de B e c, foram selecionados dados de seis postos localizados nas regiões do litoral úmido (Aracaju, Estância, Siriri), agreste (Carira, Japoatã, Tobias Barreto) e semiárido (Curalinho, Aquidabã, Samambaia) para a determinação dos parâmetros da Eq. 1 considerando-se a distribuição de Gumbel e os seguintes dados: a) utilização de toda a série histórica, desconsiderando-se a Tabela 2; b) aplicação na integra dos valores limites da Tabela 2; como resultado, têm-se os coeficientes da Tabela 5 obtidos com a série definida em (a), em todas as regiões climáticas, com os valores de B e c permanecendo os mesmos da Tabela 4. Por outro lado, para alternativa $b$ os parâmetros variaram de posto a posto.

Considerando o posto Aracaju e os coeficientes listados nas Tabelas 4 e 5 , para $T_{r}=10$ anos e $\mathrm{t}=15$ min (comuns em obras hidráulicas - CETESB (1986)) tem-se, como intensidade, respectivamente, $168,20,160,25$ e $163,77 \mathrm{~mm} \mathrm{~h}^{-1}$, ou seja, um erro de $\sim 5 \%$ entre o menor e o maior valor. Este erro pode ser considerado desprezível em face das incertezas no processo de determinação dos parâmetros e da própria série. Assim, é possível inferir que as metodologias acima citadas para obtenção dos parâmetros da Eq. 1 levam a valores com mesma ordem de grandeza.

Considerando os dados da Tabela 4, a divisão climática do Estado e a localização dos postos (Figura 1) observa-se que os valores de K para o Litoral úmido foram acima de $1000 \mathrm{em} 24$
Tabela 5. Parâmetros da equação IDF (chuvas intensas e curvas) conforme a série histórica utilizada

\begin{tabular}{|c|c|c|c|c|c|c|}
\hline \multirow{2}{*}{$\begin{array}{l}\text { Região } \\
\text { climática }\end{array}$} & \multirow{2}{*}{ Posto } & \multirow{2}{*}{ Alternativa } & \multicolumn{4}{|c|}{ Parâmetros } \\
\hline & & & K & a & B & C \\
\hline \multirow{6}{*}{ Litoral } & Aracaju & $\mathrm{a}$ & 1117,33 & 0,212 & 10,52 & 0,753 \\
\hline & Aracaju & $b$ & 1092,22 & 0,196 & 9,32 & 0,736 \\
\hline & Estância & $\mathrm{a}$ & 862,60 & 0,230 & 10,52 & 0,753 \\
\hline & Estância & $\mathrm{b}$ & 927,32 & 0,184 & 9,37 & 0,735 \\
\hline & Siriri & $\mathrm{a}$ & 857,23 & 0,218 & 10,52 & 0,753 \\
\hline & Siriri & $b$ & 972,77 & 0,163 & 10,28 & 0,749 \\
\hline \multirow[t]{6}{*}{ Agreste } & Carira & $\mathrm{a}$ & 703,11 & 0,251 & 10,52 & 0,753 \\
\hline & Carira & $b$ & 875,58 & 0,187 & 9,14 & 0,731 \\
\hline & Japoatã & $\mathrm{a}$ & 1018,12 & 0,271 & 10,52 & 0,753 \\
\hline & Japoatã & $b$ & 852,47 & 0,247 & 5,88 & 0,683 \\
\hline & Tobias Barreto & $\mathrm{a}$ & 630,09 & 0,191 & 10,52 & 0,731 \\
\hline & Tobias Barreto & $b$ & 437,92 & 0,139 & 3,82 & 0,645 \\
\hline \multirow[t]{6}{*}{ Semiárido } & Samambaia & $\mathrm{a}$ & 709,15 & 0,144 & 10,52 & 0,753 \\
\hline & Samambaia & b & 664,46 & 0,119 & 8,69 & 0,723 \\
\hline & Curralinho & $\mathrm{a}$ & 587,54 & 0,193 & 10,52 & 0,753 \\
\hline & Curralinho & b & 725,11 & 0,137 & 9,29 & 0,734 \\
\hline & Aquidabã & $\mathrm{a}$ & 949,19 & 0,168 & 13,32 & 0,791 \\
\hline & Aquidabã & b & 622,19 & 0,153 & 7,02 & 0,701 \\
\hline
\end{tabular}

postos $(50 \%$ do total) porém o maior valor deste coeficiente foi encontrado para a região do agreste $(\mathrm{K}=1357,30$; Japoatã $)$ mesmo que, para esta região, apenas em 16 postos $(33 \%$ do total) o valor de $\mathrm{K}$ ficou acima de 1000 . Para o semiárido o valor de $\mathrm{K}$ foi sempre abaixo de 980 . Por outro lado, para o coeficiente a o maior valor foi também para a região do agreste $(a=0,24$; Japoatã) muito embora na média os maiores valores de a tenham sido para o litoral úmido.

Esses dados se traduzem em chuvas muito intensas no litoral úmido, visto que, considerando a duração da chuva $t=5 \mathrm{~min}$ e para diversos períodos de retorno, a máxima de $274,07 \mathrm{~mm}$ $\mathrm{h}^{-1}$ foi para o posto Santo Amaro das Brotas e uma mínima 
Tabela 6. Valores de intensidade média para diversos períodos de retorno $\left(\mathrm{T}_{\mathrm{r}}\right)$

\begin{tabular}{|c|c|c|c|c|c|c|c|c|c|c|}
\hline Região/Duração (min) & 5 & 10 & 20 & 30 & 60 & 120 & 180 & 360 & 720 & 1440 \\
\hline $\begin{array}{l}\text { Litoral úmido } \\
\text { Máx. Int }\left(\mathrm{mm} \mathrm{h}^{-1}\right) \\
\text { Mín. Int }\left(\mathrm{mm} \mathrm{h}^{-1}\right)\end{array}$ & $\begin{array}{l}274,07 \\
167,13\end{array}$ & $\begin{array}{l}222,09 \\
135,43\end{array}$ & $\begin{array}{l}164,71 \\
100,44\end{array}$ & $\begin{array}{r}133,05 \\
81,14\end{array}$ & $\begin{array}{l}87,66 \\
53,46\end{array}$ & $\begin{array}{l}55,14 \\
33,63\end{array}$ & $\begin{array}{l}41,48 \\
25,29\end{array}$ & $\begin{array}{l}25,14 \\
15,33\end{array}$ & $\begin{array}{r}15,08 \\
9,19\end{array}$ & $\begin{array}{l}8,99 \\
5,48\end{array}$ \\
\hline $\begin{array}{l}\text { Agreste } \\
\text { Máx. Int }\left(\mathrm{mm} \mathrm{h}^{-1}\right) \\
\text { Mín. Int }\left(\mathrm{mm} \mathrm{h}^{-1}\right)\end{array}$ & $\begin{array}{l}345,42 \\
140,14 \\
\end{array}$ & $\begin{array}{l}279,91 \\
113,56 \\
\end{array}$ & $\begin{array}{r}207,59 \\
84,22 \\
\end{array}$ & $\begin{array}{r}167,69 \\
68,03 \\
\end{array}$ & $\begin{array}{r}110,49 \\
44,83 \\
\end{array}$ & $\begin{array}{r}69,50 \\
28,20 \\
\end{array}$ & $\begin{array}{l}52,27 \\
21,21 \\
\end{array}$ & $\begin{array}{l}31,68 \\
12,85 \\
\end{array}$ & $\begin{array}{r}19,00 \\
7,71 \\
\end{array}$ & $\begin{array}{r}11,34 \\
4,60 \\
\end{array}$ \\
\hline $\begin{array}{l}\text { Semiárido } \\
\text { Máx. Int }\left(\mathrm{mm} \mathrm{h}^{-1}\right) \\
\text { Mín. Int }\left(\mathrm{mm} \mathrm{h}^{-1}\right)\end{array}$ & $\begin{array}{l}196,67 \\
134,15\end{array}$ & $\begin{array}{l}159,37 \\
108,71\end{array}$ & $\begin{array}{r}118,19 \\
80,62 \\
\end{array}$ & $\begin{array}{l}95,48 \\
65,13\end{array}$ & $\begin{array}{l}62,91 \\
42,91\end{array}$ & $\begin{array}{l}39,57 \\
26,99\end{array}$ & $\begin{array}{l}29,76 \\
20,30\end{array}$ & $\begin{array}{l}18,04 \\
12,30\end{array}$ & $\begin{array}{r}10,82 \\
7,38\end{array}$ & $\begin{array}{l}6,45 \\
4,40\end{array}$ \\
\hline
\end{tabular}

das máximas foi de $167,13 \mathrm{~mm} \mathrm{~h}^{-1}$ para a Fazenda Camboatá; entretanto, as maiores intensidades máximas foram para o posto de Japoatã (Agreste) e variaram de $345,42 \mathrm{~mm} \mathrm{~h}^{-1}(\mathrm{t}=5 \mathrm{~min}$; Japoatã) a $11,34 \mathrm{~mm} \mathrm{~h}^{-1}(\mathrm{t}=24 \mathrm{~h}$; Japoatã). Por outro lado, entre os valores máximos as menores intensidades ocorrem para $\mathrm{o}$ semiárido (de 196,67 $\mathrm{mm} \mathrm{h}^{-1}(\mathrm{t}=5 \mathrm{~min}$; Aquidabã) a $6,45 \mathrm{~mm}$ $\mathrm{h}^{-1}(\mathrm{t}=24 \mathrm{~h}$; Aquidabã) (Tabela 6). Nesta tabela também se nota um decréscimo de intensidade com a duração sendo maior para o litoral. $\mathrm{O}$ resultado reflete o fato de que, quanto mais próximo do litoral maiores a intensidade e o total precipitado para dada duração.

Considerando que somente para a cidade de Aracaju existe um trabalho prévio sobre chuvas intensas (CETESB, 1986) esses dados foram utilizados para validar a equação de Aracaju, desenvolvida no presente trabalho; como resultado tem-se, como valores médios para todos os períodos de retorno, $\mathrm{R}^{2}=$ 0,996, $\mathrm{EPE}=4,610$. Ainda para $\mathrm{T}_{\mathrm{r}}=10$ anos, muito utilizado em projetos hidroagrícolas e de obras de microdrenagem urbana (Cecílio \& Pruski, 2003) os valores acima citados foram $\mathrm{R}^{2}$ $=0,996, \mathrm{EPE}=1,24$, os quais podem ser considerados bons.

Visto que não foi possível desenvolver as equações IDF para a maioria dos municípios e se considerando a variabilidade entre os parâmetros e o fato de que os dados de uma região só poderão ser utilizados para aquela região (Berne et al., 2004), efetuou-se a regionalização dos parâmetros $\mathrm{K}$ e a (obtidos da distribuição de Gumbel), via método do inverso do quadrado da distância (Cecílio \& Pruski, 2003) seguida da sua espacialização (Figuras 2 e 3 ).

Ao comparar os valores espacializados de $\mathrm{K}$ e a (Figuras 2 e 3) e o mapa das regiões climáticas do estado de Sergipe (Figura 1) percebe-se que a região do agreste sergipano e o semiárido são aquelas nas quais ocorrem os menores valores dos citados parâmetros evidenciando uma quantidade menor de precipitação, diferente do que ocorre no litoral úmido.

\section{CONClusÕes}

1. Considerando os resultados com as distribuições, houve tendência, em 44 postos, de melhor aderência das frequências dos dados à distribuição de Weibull, podendo esta ser utilizada para estudos de chuvas intensas.

2. Os parâmetros da equação IDF se correlacionaram melhor ao método de regressão não-linear.

3. A utilização da série completa levou a valores de intensidade muito próximos daqueles calculados considerandose a série limitada por valores mínimos de precipitação.

4. Os maiores valores de $\mathrm{K}$ e a foram encontrados para o litoral úmido seguido da região do Agreste.
5. Uma vez espacializados os parâmetros podem ser empregados em obras hidráulicas em locais em que não existem dados disponíveis.

\section{Agradecimentos}

À Agência Nacional das Águas e ao Instituto Nacional de Meteorologia (INMET), pelos dados de precipitação; à Secretaria de Recursos Hídricos do Estado de Sergipe; à Universidade Federal de Sergipe; à Empresa Brasileira de Pesquisa Agropecuária (EMBRAPA); à Universidade Federal de Campina Grande e à Universidade Federal de Pernambuco; à Profa. Dra. Suzana M.G. Montenegro e ao Eng. Bruno Marcionilo Silva.

\section{Literatura Citada}

ANA - Agência Nacional das Águas. Hidro Web: sistemas de informações hidrológicas. http://hidroweb.ana.gov.br. 10 Mar. 2011.

Aragão, R.; Almeida, J. A. P.; Figueiredo, E. E.; Srinivasan, V. S. Mapeamento do potencial de erosão laminar na Bacia do Rio Japaratuba, SE, via SIG. Revista Brasileira de Engenharia Agrícola e Ambiental, v.15, p.731-740, 2011.

Aragão, R.; Figueiredo, E. E.; Srinivasan, V. S.; Gois, R. S. S. Chuvas intensas no Estado da Paraíba. In: V Simpósio de Recursos Hídricos do Nordeste, 2000, Natal. Anais.. Natal: ABRH, 2000. p.74-85.

Back, A. J. Relações entre precipitações intensas de diferentes durações ocorridas no município de Urussanga, SC. Revista Brasileira de Engenharia Agrícola e Ambiental. v.13, p.170-175, 2009.

Ben-Zvi, A. Rainfall intensity-duration-frequency relationships derived from large partial duration series. Journal of Hydrology, v.367, p.104-114, 2009.

Berne, A.; Delrieu, G.; Creutin, J. D.; Obled, C. Temporal and spatial resolution of rainfall measurements required for urban hydrology. Journal of Hydrology, v.299, p.166-179, 2004.

Cecílio, R. A.; Pruski, F. F. Interpolação dos parâmetros da equação de chuvas intensas com uso do inverso de potências da distância. Revista Brasileira de Engenharia Agrícola e Ambiental, v.7, p.501-504, 2003.

CETESB - Companhia de Tecnologia de Saneamento Ambiental. Drenagem urbana: manual de projeto. 1.ed. São Paulo: DAEE/CETESB, 1986. 466p. 
Damé, R. C. F.; Teixeira, C. F. A.; Terra, V. S. S.; Rosskoff, J.L.C. Hidrograma de projeto em função da metodologia utilizada na obtenção da precipitação. Revista Brasileira de Engenharia Agrícola e Ambiental, v.14, p.46-54, 2010.

Garcia, S. S.; Amorim, R. S. S.; Couto, E. G.; Stopa, W. H. Determinação da equação intensidade-duração-frequência para três estações meteorológicas do Estado de Mato Grosso. Revista Brasileira de Engenharia Agrícola e Ambiental, v.15, p.575-581, 2011.

Grimm. A. M. Interannual climate variability in South America: Impacts on seasonal precipitation, extreme events, and possible effects of climate change. Stoch Environ Reserch Risk Assess, v.25, p.537-554, 2011.

IBGE - Instituto Brasileiro de Geografia e Estatística. Sinopse do censo demográfico 2010. Estimativas/Contagem da População, Rio de Janeiro: IBGE, 2011. 261p.

Lambert, F. H.; Stine, A. R.; Krakauer, N. Y.; Chiang, J. C. H. How much will precipitation increase with global warming? EOS, Transactions American Geophysical Union, v.89, p.193-200. 2008.

MCT/CGE - Ministério de Ciência e Tecnologia/Centro de Estudos e Gestão Estratégica. Diretrizes estratégicas para o Fundo de Recursos Hídricos de Desenvolvimento Científico e Tecnológico. Brasília, 2002. http://www.ana.gov.br, 11 Out. 2011.

Mello, C. R.; Silva, A. M.; Lima, J. M.; Ferreira, D. F.; Oliveira, M. S. Modelos matemáticos para predição da chuva de projeto para regiões do Estado de Minas Gerais. Revista Brasileira de Engenharia Agrícola e Ambiental, v.7, p.121-128, 2003.

Mendes, H. C.; Mendiondo, E. M. Histórico da expansão urbana e Incidência de Inundações: O Caso da Bacia do Gregório, São Carlos - SP. Revista Brasileira de Recursos Hídricos. v.12, p.17-27, 2007.

Min, S.; Zhang, X.; Zwiers; F. W.; Heger, G. C. Human contribution to more-intense precipitation extremes. Nature. Letter. v.470, p.378, 2011.

Murthy, D. N. P, Xie, M. Jiang, R. Weibull models, Wiley series in probability and statistics, New Jersey: John Wiley \& Sons, 2004. 396p.

Naghettini, M.; Pinto, E. J. A. Hidrologia estatística. Belo Horizonte: CPRM. 2007. 552p.
Oliveira, L. F. C.; Antonini, J. C. A.; Fioreze, A. P.; Silva, M. A. S. Métodos de estimativa de precipitação máxima para o Estado de Goiás. Revista Brasileira de Engenharia Agrícola e Ambiental v.12, p.620-625, 2008.

Overeem, A.; Buishand, A.; Holleman, I. Rainfall depthduration-frequency curves and their uncertainties. Journal of Hydrology, v.348, p.124-134, 2008.

Sansigolo, C. A. Distribuições de extremos de precipitação diária, temperatura máxima e mínima e velocidade do vento em piracicaba, SP (1917-2006). Revista Brasileira de Meteorologia, v.23, p.341-346, 2008.

Santos, G. S.; Nori, P. G. Oliveira, L. F. C. Chuvas intensas relacionadas à erosão hídrica. Revista Brasileira de Engenharia Agrícola e Ambiental, v.14, p.115-123, 2010.

SEMARH - Secretaria de Meio Ambiente e Recursos Hídricos de Sergipe. Atlas digital de recursos hídricos do Estado de Sergipe. Aracaju: SEMARH, 2011. CD Rom

Silva, B. M. Chuvas intensas em localidades do estado de Pernambuco. Recife: UFPE, 2009. 100p. Dissertação Mestrado

Silva, D. D.; Gomes Filho, R. R.; Pruski, F. F.; Pereira, S. B.; Novaes, L. F. Chuvas intensas no Estado da Bahia. Revista Brasileira de Engenharia Agrícola e Ambiental, v.6, p.362367, 2002.

Silva, W. P. da; Silva, C. M. D. P. S. e; Cavalcanti, C. G. B.; Diogo D. P. S. e; Silva, I. B. S. Lab-fit ajuste de curvas: Um software em português para tratamento de dados experimentais. Revista Brasileira de Ensino de Física, v.26, p.419-427, 2004.

Svensson, C.; Clarke, R. T.; Jones, D. A. An experimental comparison of methods for estimating rainfall intensityduration-frequency relations from fragmentary records. Journal of Hydrology, v.341, p.79-89, 2007.

Teixeira, C. F. A.; Damé, R. de C. F.; Siqueira, G. do A.; Bacelar, L. C. S. D. Vazão máxima de projeto: Metodologia para dimensionamento de bueiros em áreas agrícolas. Teoria e Prática na Engenharia Civil, n.17, p.49-56, 2011.

Wei, W.; Chen, L.; Fu, B.; Huang, Z.; Wu, D.; Gui, L. The effect of land uses and rainfall regimes on runoff and soil erosion in the semi-arid loess hilly area, China. Journal of Hydrology, v.335, p.247-258, 2007. 